\title{
3D-3D Tubular Organ Registration and Bifurcation Detection from CT Images
}

\author{
Jinghao Zhou ${ }^{1}$, Sukmoon Chang ${ }^{2}$, Dimitris Metaxas ${ }^{3}$ and Gig Mageras ${ }^{4}$ \\ ${ }^{1}$ Department of Radiation Oncology \\ The Cancer Institute of New Jersey \\ Robert Wood Johnson Medical School \\ University of Medicine and Dentistry of New Jersey \\ USA \\ ${ }^{2}$ Computer Science, Capital College, Pennsylvania State University \\ USA \\ ${ }^{3}$ CBIM, Rutgers University \\ USA \\ ${ }^{4}$ Department of Medical Physics \\ Memorial Sloan-Kettering Cancer Center
}

USA

\section{Introduction}

The registration of tubular organs (pulmonary tracheobronchial tree or vasculature) of 3D medical images is critical in various clinical applications such as surgical planning and radiotherapy. For example, the pulmonary tracheobronchial tree or vascular structures can be used as the landmarks in lung tumor resection planning; the quantifying treatment effectiveness of the radiotherapy on lung nodules is based on the registration of the pulmonary tracheobronchial tree or vessels; the planning inter-patients partial liver transplants use registered contrast injection angiography (CTA) to create digital-subtraction contrast injection angiography (CTA) of liver vessels. The bifurcation of the tubular organs plays a critical role in clinical practices as well. Inflammation caused by bronchitis alters the airway branching configuration which causes various breathing problems (Luo et al., 2007). Atherosclerotic disease at the bifurcation has been widely known as a risk factor for cerebral ischemic episodes and infarction (Binaghi et al., 2001). The bifurcation points (or the branching points) have been chosen to build the validation protocol of the registration methods (Gee et al., 2002).

Many researchers have developed various methods for registration of tubular organs from medical images. Baert et al. (2004) used an intensity based 2D-3D registration algorithm to register the pre-operative 3D Magnetic Resonance Angiogram (MRA) data to the interventional digital subtraction angiography (DSA) images. Chan et al. (2004) proposed a 2D-3D vascular registration algorithm based on minimizing the sum of squared differences between the projected image and the reference DSA image. However, these registration methods are all developed for applications with 2D-3D registration. Chan \& Chung (2003) solves a 3D$3 \mathrm{D}$ registration problem by transform the problem into 2D-3D registration problem. Aylward 
et al. (2003) presented a registration method by registering a model of the tubes in the source image directly with the target images. This method extracted an accurate model of the tubes in the source image and multiple target images without extractions could be registered with that model. However, this method does not utilize the information in the bifurcation points of the tubular organs.

In this chapter, we present a rigid registration method of the tubular organs based on the automatically detected bifurcation points of the tubular organs. There are two steps in our approach. We first perform a 3D tubular organ segmentation method to extract the centerlines of tubular organs and radius estimation in both planning and respiration-correlated CT images. This segmentation method automatically detects the bifurcation points by applying Adaboost algorithm with specially designed filters. We then apply a rigid registration method which minimizes the least square error of the corresponding bifurcation points between the planning CT images and the respiration-correlated CT (RCCT) images.

\section{Method}

Our method consists of two steps: the first step is the 3D tubular organ segmentation method to extract the centerlines of tubular organs in both planning and respiration-correlated CT images with the analysis of the Hessian matrix and bifurcation detection using Adaboost with specially designed filters (Zhou et al., 2007); in the second step, we apply a rigid registration method which minimizes the least square error of the corresponding bifurcation points between the planning and respiration-correlated CT images. Without loss of generality, we assume that the tubular organs appear brighter than the background and their centerlines coincide with the ridges in the intensity profile. When the vessel tree is segmented, the original CT images will be used. When the pulmonary tracheobronchial tree is segmented, the inverted CT images will be used.

\subsection{Tubular organ segmentation and bifurcation detection}

\subsubsection{Tubular organ direction estimation and normal plane extraction}

The eigenanalysis of the Hessian matrix is a widely used method for tubular organs detection (Danielsson \& Lin, 2001; Lorenz et al., 1997; Zhou et al., 2006). The signs and ratios of the eigenvalues provide the indications of various shapes of interest, as summarized in Table 1. Also, the eigenvector corresponding to the largest eigenvalue can be used as an indicator of the elongated direction of tubular organs.

Given an image $I(x)$, the local intensity variations in the neighborhood of a point $x_{0}$ can be expressed with its Taylor expansion:

$$
I\left(x_{0}+h\right) \approx I\left(x_{0}\right)+h^{T} \nabla I\left(x_{0}\right)+h^{T} H\left(x_{0}\right) h
$$

\begin{tabular}{|c|c|}
\hline Eigenvalues & Shape \\
\hline \hline$\lambda_{1} \leq 0, \lambda_{2} \leq 0, \lambda_{3} \leq 0$ & blob \\
\hline$\lambda_{1} \leq 0, \lambda_{2} \leq 0, \lambda_{3} \approx 0$ & tube \\
\hline$\lambda_{1} \leq 0, \lambda_{2} \approx 0, \lambda_{3} \approx 0$ & plane \\
\hline$\lambda_{1} \leq 0, \lambda_{2} \leq 0, \lambda_{3} \geq 0$ & double cone \\
\hline
\end{tabular}

Table 1. Criteria for eigenvalues and corresponding shapes. 


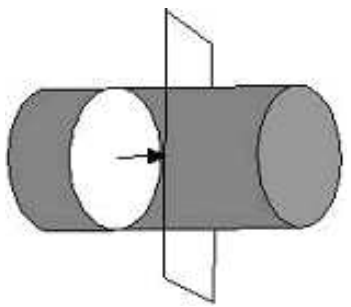

Fig. 1. Tracing along the direction of the tubular organs. Figure shows $\overrightarrow{e_{3}}$ and the normal plane defined by $\overrightarrow{e_{1}}$ and $\overrightarrow{e_{2}}$.

where, $\nabla I\left(x_{0}\right)$ and $H\left(x_{0}\right)$ denote the gradient and the Hessian matrix of $I$ at $x_{0}$, respectively. Let $\lambda_{1}, \lambda_{2}, \lambda_{3}$ and $\overrightarrow{e_{1}}, \overrightarrow{e_{2}}, \overrightarrow{e_{3}}$ be the eigenvalues and eigenvectors of $H$ such that $\lambda_{1} \leq \lambda_{2} \leq \lambda_{3}$ and $\left|\vec{e}_{i}\right|=1$.

Tracing the centerlines of tubular organs by integrating along the elongated direction of tubular organs may be less sensitive to image noise (Aylward \& Bullitt, 2002). Our method for tracing the centerlines of tubular organs starts from a preselected point (and, thereafter, from the point selected in the previous step) and follows the estimated direction of tubular organs to extract intensity ridges. The intensity ridges in 3D must meet the following constraints:

$$
\begin{gathered}
\lambda_{1} \ll 0, \quad \lambda_{2} \ll 0 \\
\overrightarrow{e_{1}} \cdot \nabla I(x) \approx 0 \text { and } \overrightarrow{e_{2}} \cdot \nabla I(x) \approx 0
\end{gathered}
$$

Note that the intensity reduces away from the ridge: $\lambda_{1} / \lambda_{2} \approx 0$. Also note that the ridge point must be a local maximum of the plane defined by $\overrightarrow{e_{1}}$ and $\overrightarrow{e_{2}}$, while $\overrightarrow{e_{3}}$ is normal to the plane. Thus, $\overrightarrow{e_{1}}$ and $\overrightarrow{e_{2}}$ define the cross-sectional plane orthogonal to the tubular organs, while $\overrightarrow{e_{3}}$ provides the estimate of the tubular organs direction. Therefore, to trace tubular organs centerlines, the cross-sectional plane defined by $\overrightarrow{e_{1}}$ and $\overrightarrow{e_{2}}$ is shifting a small step along the direction of the tubular organs given by $\overrightarrow{e_{3}}$ (Fig. 1).

\subsubsection{Bifurcation detection using AdaBoost}

Boosting is a method for improving the performance of any weak learning algorithm which, in theory, only needs to perform slightly better than random guessing. A boosting algorithm called AdaBoost improves the performance of a given weak learning algorithm by repeatedly running the algorithm on the training data with various distributions and then combining the classifiers generated by the weak learning algorithm into a single final classifier (Freund \& Schapire, 1996; Schapire, 2002). The proposed method uses AdaBoost with specially designed filters for fully automatic detection of bifurcation points.

We design three types of linear filters to capture the local appearance characteristics: 2D Gaussian filters to capture low frequency information; the first order derivatives of 2D Gaussian filters to capture high frequency information, i.e., edges; the second order derivatives of 2D Gaussian filters to capture local maxima, i.e., ridges (Lindeberg, 1999). These filters function as weak classifiers for AdaBoost.

We design three types of linear filters to capture the local appearance characteristics: 2D Gaussian filters to capture low frequency information; the first order derivatives of 2D Gaussian filters to capture high frequency information, i.e., edges; the second order derivatives of $2 \mathrm{D}$ 


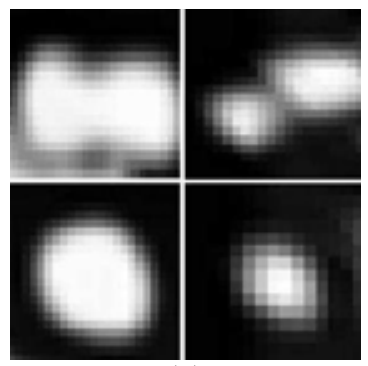

(a)

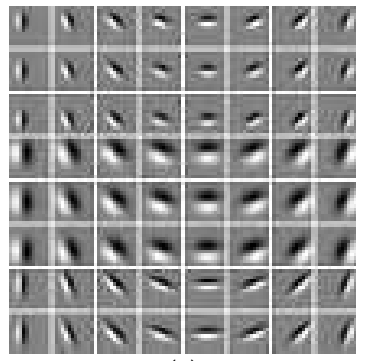

(c)

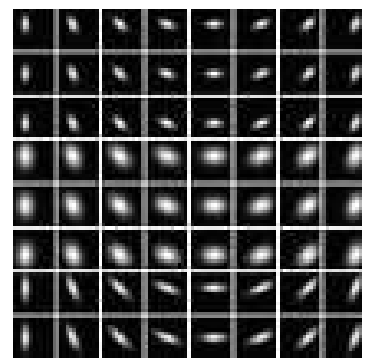

(b)

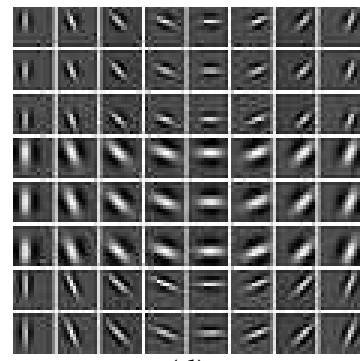

(d)

Fig. 2. (a) The cross-sectional planes of the pulmonary tracheobronchial tree with bifurcation (top row) and without bifurcation (bottom row), (b) 2D Gaussian used for low frequency information detection, (c) the first derivatives of Gaussian used for edge detection, and (d) the second derivatives of Gaussian used for ridge detection.

Gaussian filters to capture local maxima, i.e., ridges (Lindeberg, 1999). These filters function as weak classifiers for AdaBoost.

Let $G=G\left(\mu_{x}, \mu_{y}, \sigma_{x}, \sigma_{y}, \theta\right)$ be an asymmetric 2D Gaussian, where

$$
\left(\begin{array}{l}
\mu_{x} \\
\mu_{y}
\end{array}\right)=R \times\left(\begin{array}{l}
x-x_{0} \\
y-y_{0}
\end{array}\right), \quad R=\left(\begin{array}{cc}
\cos \theta & -\sin \theta \\
\sin \theta & \cos \theta
\end{array}\right)
$$

and, $\left(\sigma_{x}, \sigma_{y}\right),\left(x_{0}, y_{0}\right)$, and $\theta$ are the standard deviation, translation, and rotational parameters of $G$, respectively. We set the derivatives of $G$ to have the same orientation as $G$ :

$$
\begin{gathered}
G^{\prime}=G_{x} \cos (\theta)+G_{y} \sin (\theta) \\
G^{\prime \prime}=G_{x x} \cos ^{2}(\theta)+2 \cos (\theta) \sin (\theta) G_{x y}+G_{y y} \sin ^{2}(\theta)
\end{gathered}
$$

From the above equations, we tune $x_{0}, y_{0}, \sigma_{x}, \sigma_{y}$, and $\theta$ to generate the desired filters. For a $15 \times 15$ sized window, we designed the total of 16,200 filters $-x_{0} \times y_{0} \times\left(\sigma_{x}, \sigma_{y}\right) \times \theta=$ $10 \times 10 \times 3 \times 18=5,400$ filters for each of $G, G^{\prime}$, and $G^{\prime \prime}$. Some of the filter are shown in Fig. 2.

We then normalized the cross-sectional planes obtained from the previous step to the size of the filters and collected an example set containing both positive (i.e., samples with bifurcation) and negative (i.e., samples without bifurcation) examples from the normalized planes. The AdaBoost method is used to classify positive training examples from negative examples by 
selecting a small number of critical features from a huge feature set previously designed and creating a weighted combination of them to use as a strong classifier. Even when the strong classifier consists of a large number of individual features, AdaBoost encounters relatively few overfitting problems (Viola \& Jones, 2001).

During the boosting process, every iteration selects one feature from the entire feature set and combines it with the existing classifier obtained from previous iterations. After a sufficient number of iterations, the weighted combination of the selected features become a strong classifier with high accuracy. That is, the output of the strong classifier is the weighted sum of the outputs of the selected features (i.e., weak classifiers): $F=\sum_{t} \alpha_{t} h_{t}(x)$, where $\alpha_{t}$ and $h_{t}$ are weights and outputs of weak classifiers, respectively. We call $F$ the bifurcation criterion. AdaBoost classifies an example plane as a sample with bifurcation when $F>0$ and as a sample without bifurcation when $F<0$.

To estimate the generalization error of AdaBoost in classification, we applied bootstrapping (Efron, 1983). We trained and tested the method on a bootstrap sample, i.e., a sample of size $m$ chosen uniformly at random with replacement from the original example set of size $m$. the test error continues improving even after the training error has already become zero and converges to error rate of $3.9 \%$ after about 20 iterations of boosting steps, i.e., $95 \%$ confidence interval of $3.1 \sim 4.6 \%$.

\subsubsection{Tubular organs radius estimation for 3D reconstruction}

We use a deformable sphere model to estimate the radii of the tubular organs for 3D tubular organs reconstruction (Zhou et al., 2007). At each of the detected center points as well as the detected branching points, a deformable sphere is initialized. The position of points on the model are given by a vector-valued, time varying function of the model's intrinsic coordinates $\vec{u}:$

$$
\vec{x}(\vec{u}, t)=\left(x_{1}(\vec{u}, t), x_{2}(\vec{u}, t), x_{3}(\vec{u}, t)\right)^{T}=\vec{c}(t)+\vec{R}(t) \vec{s}(\vec{u}, t)
$$

where, $\vec{c}(t)$ is the origin of a noninertial, model-centered reference frame $\Phi, \vec{R}(t)$ is the rotation matrix for the orientation of $\Phi$, and $\vec{s}(\vec{u}, t)$ denotes the positions of points on the reference shape relative to the model frame Metaxas (1997). The reference shape of a sphere is generated in spherical coordinate system with fixed intervals along longitude and latitude directions in the parametric $(u, v)$ domain:

$$
\vec{e}(u, v)=\left(\begin{array}{l}
x \\
y \\
z
\end{array}\right)=a_{0} \cdot\left(\begin{array}{l}
a_{1} \cdot \cos u \cdot \cos v \\
a_{2} \cdot \cos u \cdot \sin v \\
a_{3} \cdot \sin u
\end{array}\right)
$$

where, $a_{0} \geq 0$ is a scale parameter and $0 \leq a_{1}, a_{2}, a_{3} \leq 1$ are deformation parameters that control the aspect ratio of the cross section of the sphere. We collected the parameters in $\vec{e}(u, v)$ into the parameter vector

$$
\vec{q}_{s}=\left(a_{0}, a_{1}, a_{2}, a_{3}\right)^{T}
$$

The velocity of a point on the model is

$$
\dot{\vec{x}}=\dot{\vec{c}}+\dot{\vec{R}} \vec{s}+\vec{R} \dot{\vec{s}}=\dot{\vec{c}}+\vec{B} \dot{\vec{\theta}}+\vec{R} \dot{\vec{s}}=\left[\begin{array}{lll}
\vec{I} & \vec{B} & \vec{R} J
\end{array}\right] \dot{\vec{q}}=\vec{L} \dot{\vec{q}}
$$

where, $\vec{\theta}$ is the vector of rotational coordinates of the model, $\vec{B}=[\partial(\vec{R} s) / \partial \vec{\theta}], \vec{J}=\left[\partial \vec{s} / \partial \vec{q}_{s}\right]$, $\vec{q}=\left(\vec{q}_{c}^{T}, \vec{q}_{\theta}^{T}, \vec{q}_{s}^{T}\right)^{T}, \vec{q}_{c}=\vec{c}, \vec{q}_{\vec{\theta}}=\vec{\theta}$, and $\vec{L}$ is the model's Jacobian matrix that maps generalized 
coordinates $\vec{q}$ into 3D vectors. When initialized near a vessel, the model deforms to fit to the vessel due to the overall forces exerted from the edge of the vessel and comes to rest when $\vec{q}$ is found that minimizes the simplified Lagrangian equation of motion:

$$
\vec{q}=\vec{f}_{\vec{q}}=\int \vec{L}^{T} \vec{f} d u
$$

where, $\vec{f}_{\vec{q}}$ is the generalized external forces associated with the degrees of freedom $\vec{q}$ of the model and $\vec{f}$ is the external force exerted from the images. In this paper, we use Gradient Vector Flow (GVF) field computed from the images as the external force (Xu \& Prince, 1998).

\subsection{Tubular organs registration}

The registration is formulated as a rigid global deformation. We denote the bifurcation points in the planning CT images as the source points and the corresponding bifurcation points in the respiration-correlated CT images as the target points. Since our tubular organs tracing starts from preselected points, the correspondence between the source points and the target points can be easily determined. The global deformation is a transformation of a point $\vec{x}$ in the planning CT image coordinate system into a point $\overrightarrow{x^{\prime}}$ in the respiration-correlated CT image coordinate system, that is, $\overrightarrow{x^{\prime}}=\vec{M} \cdot \vec{x}$, where $\vec{M}$ is the transformation matrix. Let $\vec{X}^{P}$ and $\vec{X}^{B}$ be the bifurcation points for the planning $\mathrm{CT}$ images and respiration-correlated CT images, respectively. The global deformation of $\vec{X}^{P}$ onto $\vec{X}^{B}$ is achieved by finding the parameters of a $3 \mathrm{D}$ transformation that minimizes the least square error:

$$
\varepsilon=\sum_{i=1}^{n}\left\|\vec{x}_{i}^{B}-\vec{M} \cdot \vec{x}_{i}^{P}\right\|^{2}
$$

where, $\vec{x}_{i}$ is the $i$-th point of a deformable model in the homogeneous coordinate system. We use Levenberg-Marquardt optimization method with the following Jacobian of the transformation as the metric to provide transformation parameter gradients:

$$
\frac{\partial \varepsilon}{\partial \vec{M}}=-\sum_{i=1}^{n} 2\left(\vec{x}_{i}^{B}-\vec{M} \cdot \vec{x}_{i}^{P}\right)\left(\vec{x}_{i}^{P}\right)^{T}
$$

\section{Results}

We applied our method on clinical lung CT data from six different patients. Each patient has one planning CT data set and ten respiration-correlated CT (RCCT) data sets taken in one complete respiratory cycle. They represent CT images at ten different points in the patient's breathing cycle. The number of slices in each CT scan ranged from 83 to 103 with $2.5 \mathrm{~mm}$ slice thickness(and also digitally resliced to obtain cubic voxels, resulting in 206 to 256 slices), each of which are of size $512 \times 512$ pixels, with in-plane resolution of $0.9 \mathrm{~mm}$. All experiments were performed on a $\mathrm{PC}$ with $2.0 \mathrm{GHz}$ processor and 2.0GB of memory. We first extracted 507 cross-sectional planes from the VOIs using cross-sectional plane extraction method. The extracted planes were originally of size $30 * 30$ pixels and were normalized to be the same size as the filter, i.e., $15^{*} 15$ pixels. The smallest diameter of bronchi in our samples was 3 pixels. These example planes contained 250 positive (i.e., with bifurcation) and 257 negative (i.e., without bifurcation) examples. Our method was trained with 150 positive and 150 negative examples and tested on 100 positive and 107 negative examples. We performed bootstrapping 


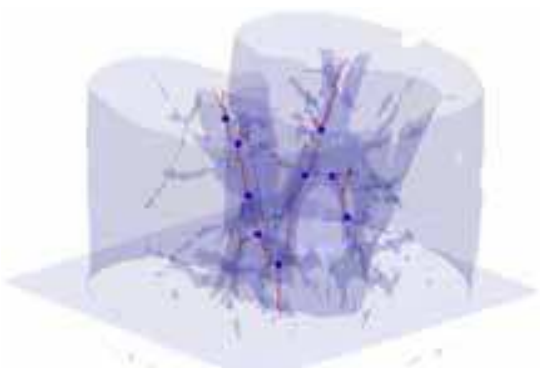

(a)

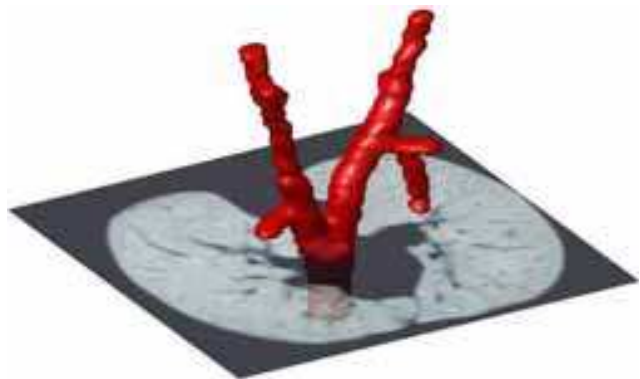

(b)

Fig. 3. Pulmonary tracheobronchial tree segmentation and bifurcation detection. (a) centerlines superimposed on an isosurface of the initial image, (b) 3D reconstruction of pulmonary tracheobronchial tree from the graph representation in (a). Blue points in (a) shows the bifurcation points detected by our method.

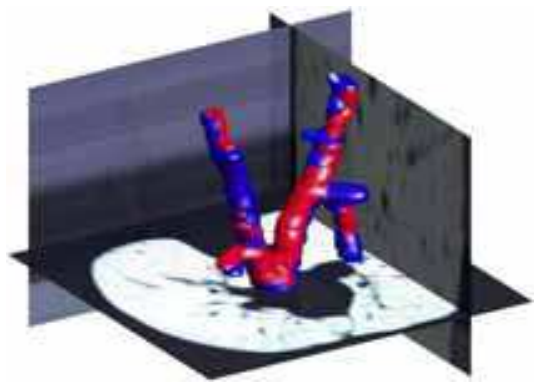

(a)

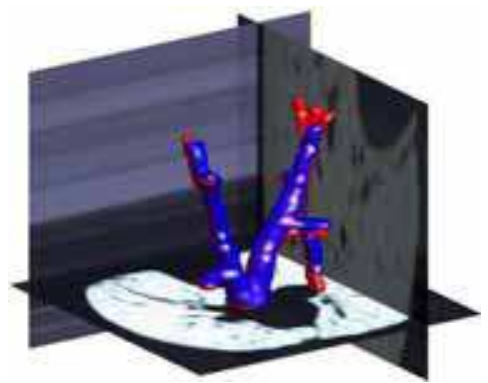

(b)

Fig. 4. Registration results. Blue shows the 3D reconstruction of pulmonary tracheobronchial tree in the registered planning images and red shows 3D reconstruction of pulmonary tracheobronchial tree in the respiration-correlated images.

to estimate the generalization error of our method, obtaining the mean error rate of $3.1 \sim 4.6 \%$, which is $95 \%$ confidence interval, as described in previous section.

Fig. 3 illustrates further visual validation of our segmentation method applied to the pulmonary tracheobronchial structures. In Fig. 3(a), the extracted centerlines are superimposed on the isosurface of the original CT images along with the detected bifurcation points by the Adaboost learning method (shown in blue). Fig. 3(b) shows the 3D reconstruction of the pulmonary tracheobronchial tree from the centerlines and bifurcation points in Fig. 3(a). Fig. 4 shows the registration results. The results are also summarized in Table 2. It shows that, on average, the mean distance and the root-mean-square error (RMSE) of the corresponding bifurcation points between the respiration-correlated images and the registered planning images are less than $2.7 \mathrm{~mm}$. There are breathing-induced deformations in the tracheobronchial tree, owing to the different amount of lung inflation in the different RCCT data sets. These may partly explain the mean distance and the root-mean-square error (RMSE) in Table 2. 


\begin{tabular}{|c||c|c|}
\hline Dataset & Mean distance $(\mathrm{mm})$ & Root mean square error $(\mathrm{mm})$ \\
\hline \hline Best & 1.51 & 1.63 \\
\hline Worst & 3.08 & 3.38 \\
\hline Average & 2.17 & 2.63 \\
\hline
\end{tabular}

Table 2. Results of the registration method on clinical datasets.

\section{Conclusion}

In this chapter, we present a novel method for tubular organs registration based on the automatically detected bifurcation points of the tubular organs. We first perform a 3D tubular organ segmentation method to extract the centerlines of tubular organs and radius estimation in both planning and respiration-correlated CT images. This segmentation method automatically detects the bifurcation points by applying Adaboost algorithm with specially designed filters. We then apply a rigid registration method which minimizes the least square error of the corresponding bifurcation points between the planning CT images and the respirationcorrelated CT images. Our method has over $96 \%$ success rate for detecting bifurcation points. We present bery promising results of our method applied to the registration of the planning and respiration-correlated $\mathrm{CT}$ images. On average, the mean distance and the rootmean-square error (RMSE) of the corresponding bifurcation points between the respirationcorrelated images and the registered planning images are less than $2.7 \mathrm{~mm}$.

\section{References}

Aylward, S. \& Bullitt, E. (2002). Initialization, noise, singularities, and scale in height ridge traversal for tubular object centerline extraction, IEEE Transactions on Medical Imaging 21(2): 61-75.

Aylward, S., Jomier, J., Weeks, S. \& Bullitt, E. (2003). Registration and analysis of vascular images, International Journal of Computer Vision 55: 123-138.

Baert, S., Penney, G., van Walsum, T. \& Niessen, W. (2004). Precalibration versus 2d-3d registration for $3 \mathrm{~d}$ guide wire display in endovascular interventions, MICCAI 3217: 577584 .

Binaghi, S., Maeder, P., Uské, A., Meuwly, J.-Y., Devuyst, G. \& Meuli, R. (2001). Threedimensional computed tomography angiography and magnetic resonance angiography of carotid bifurcation stenosis, European Neurology 46: 25-34.

Chan, H. \& Chung, A. (2003). Efficient 3d-3d vascular registration based on multiple orthogonal 2d projections, Biomedical Image Registration 2717: 301-310.

Chan, H., Chung, A., Yu, S. \& Wells, W. (2004). 2d-3d vascular registration between digital subtraction angiographic (dsa) and magnetic resonance angiographic (mra) images, IEEE International Symposium on Biomedical Imaging pp. 708-711.

Danielsson, P.-E. \& Lin, Q. (2001). Efficient detection of second-degree variations in $2 \mathrm{~d}$ and $3 \mathrm{~d}$ images, Journal of Visual Communication and Image Representation 12: 255-305.

Efron, B. (1983). Estimating the error rate of a prediction rule: Improvement on crossvalidation, Journal of the American Statistical Association 78: 316-331.

Freund, Y. \& Schapire, R. (1996). Experiments with a new boosting algorithm, the $13^{\text {th }}$ International Conference on Machine Learning, pp. 148-156. 
Gee, J., Sundaram, T., Hasegawa, I., Uematsu, H. \& Hatabu, H. (2002). Characterization of regional pulmonary mechanics from serial mri data, MICCAI pp. 762-769.

Lindeberg, T. (1999). Principles for automatic scale selection, in B. J. et al. (ed.), Handbook on Computer Vision and Applications, Academic Press, Boston, USA, pp. 239-274.

Lorenz, C., Carlsen, I.-C., Buzug, T. M., Fassnacht, C. \& Weese, J. (1997). Multi-scale line segmentation with automatic estimation of width, contrast and tangential direction in 2d and 3d medical images, CVPRMed-MRCAS, pp. 233-242.

Luo, H., Liu, Y. \& Yang, X. (2007). Particle deposition in obstructed airways, Journal of Biomechanics 40: 3096-3104.

Metaxas, D. N. (1997). Physics-Based Deformable Models: Applications to Computer Vision, Graph$i c s$ and Medical Imaging, Kluwer Academic Publishers.

Schapire, R. (2002). The boosting approach to machine learning: An overview, MSRI Workshop on Nonlinear Estimation and Classification.

Viola, P. \& Jones, M. (2001). Robust real-time object detection, Second International Workshop on Statistical and Computational Theories of Vision-Modeling, Learning, and Sampling.

Xu, C. \& Prince, J. (1998). Snakes, shapes, and gradient vector flow, IEEE Transactions on Image Processing 7(3): 359-369.

Zhou, J., Chang, S., Metaxas, D. \& Axel, L. (2006). Vessel boundary extraction using ridge scan-conversion and deformable model, IEEE International Symposium on Biomedical Imaging pp. 189-192.

Zhou, J., Chang, S., Metaxas, D. \& Axel, L. (2007). Vascular structure segmentation and bifurcation detection, IEEE International Symposium on Biomedical Imaging pp. 872-875. 


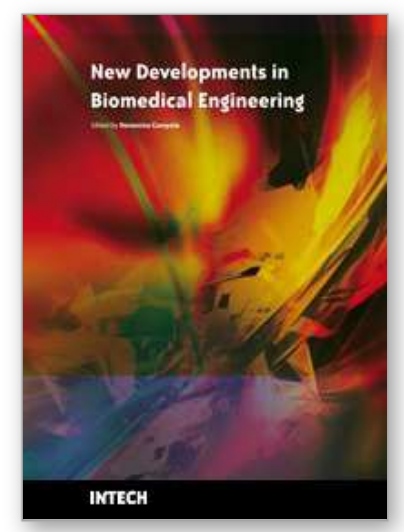

\author{
New Developments in Biomedical Engineering \\ Edited by Domenico Campolo
}

ISBN 978-953-7619-57-2

Hard cover, 714 pages

Publisher InTech

Published online 01, January, 2010

Published in print edition January, 2010

Biomedical Engineering is a highly interdisciplinary and well established discipline spanning across engineering, medicine and biology. A single definition of Biomedical Engineering is hardly unanimously accepted but it is often easier to identify what activities are included in it. This volume collects works on recent advances in Biomedical Engineering and provides a bird-view on a very broad field, ranging from purely theoretical frameworks to clinical applications and from diagnosis to treatment.

\title{
How to reference
}

In order to correctly reference this scholarly work, feel free to copy and paste the following:

Jinghao Zhou, Sukmoon Chang, Dimitris Metaxas and Gig Mageras (2010). 3D-3D Tubular Organ Registration and Bifurcation Detection from CT Images, New Developments in Biomedical Engineering, Domenico Campolo (Ed.), ISBN: 978-953-7619-57-2, InTech, Available from: http://www.intechopen.com/books/newdevelopments-in-biomedical-engineering/3d-3d-tubular-organ-registration-and-bifurcation-detection-from-ctimages

\section{INTECH}

open science | open minds

\section{InTech Europe}

University Campus STeP Ri

Slavka Krautzeka 83/A

51000 Rijeka, Croatia

Phone: +385 (51) 770447

Fax: +385 (51) 686166

www.intechopen.com

\section{InTech China}

Unit 405, Office Block, Hotel Equatorial Shanghai No.65, Yan An Road (West), Shanghai, 200040, China 中国上海市延安西路65号上海国际贵都大饭店办公楼 405 单元 Phone: +86-21-62489820

Fax: $+86-21-62489821$ 
(C) 2010 The Author(s). Licensee IntechOpen. This chapter is distributed under the terms of the Creative Commons Attribution-NonCommercial-ShareAlike-3.0 License, which permits use, distribution and reproduction for non-commercial purposes, provided the original is properly cited and derivative works building on this content are distributed under the same license. 